\title{
Avaliação da fisioterapia sobre o equilíbrio e a qualidade de vida em pacientes com esclerose múltipla
}

\author{
Evaluation of the physical therapy on the balance and on the quality of life in multiple \\ sclerosis patients
}

\author{
Iclaikouski Farias Rodrigues ${ }^{1}$, Mariângela Braga Pereira Nielson², Andresa \\ Rosane Marinho ${ }^{3}$
}

\section{RESUMO}

Introdução. A Esclerose Múltipla (EM) é uma doença desmielinizante do SNC. Os principais distúrbios são fadiga intensa, déficit de equilíbrio e incoordenação motora. Objetivos. Avaliar os efeitos da fisioterapia sobre o equilíbrio e a qualidade de vida dos pacientes com EM. Métodos. O estudo foi do tipo intervencional analítico, onde 20 indivíduos foram aleatorizados em 2 grupos: Grupo 1 - submetidos a 15 intervenções fisioterapêuticas específicas para EM, sendo 3 vezes por semana; Grupo 2 - realizaram fisioterapia convencional uma vez na semana. Foi realizada análise descritiva dos dados através de tabelas com média e desvio padrão. Para comparação intra e inter-grupos foi utilizado o teste T de Student. Resultados. Foram avaliados 20 sujeitos, onde 85\% (17) eram do sexo feminino e $15 \%$ (3) do sexo masculino, com média de idade de $40 \pm 12$ anos, variando entre 18 a 63 anos. Houve melhora do equilíbrio $(\mathrm{p}=$ $0,011)$ e da qualidade de vida $(p=0,006)$, após a intervenção fisioterapêutica específica. Já no grupo convencional não houve melhora significativa. Conclusão. O equilíbrio e a qualidade de vida dos indivíduos com EM melhoraram significativamente sob intervenção fisioterapêutica direcionada.

Unitermos: Fisioterapia. Esclerose Múltipla. Equilíbrio Musculosquelético. Qualidade de Vida.

Citação: Rodrigues IF, Nielson MBP, Marinho AR. Avaliação da fisioterapia sobre o equilíbrio e a qualidade de vida em pacientes com esclerose múltipla.

\section{SUMMARY}

Introduction. Multiple Sclerosis (MS) is a CNS demyielin disease. The main disturbs are intense fatigue, balance disturb and ataxy. Objectives. To evaluate the physical therapy effects on the balance and quality of life of MS patients. Methods. This study was analytic and interventional, the patients were randomized in two groups: Group 1: submitted in a fifteen sessions of physical therapy program, three times per week; and Group 2: participated in a conventional physical therapy program once a week. The data descriptive analyze was made through tables with mean and standard deviation. The comparison before and after physical therapy program and between groups was made by Student t test. Results. Twenty subjects was evaluated, $85 \%(17)$ were female and $17 \%$ (3) were male, with mean age $40 \pm 12$ years old, ranging from 18 to 63 years old. There was significant improvement in balance $(p=0.011)$ and quality of life $(p=0.006)$ after specific physical therapy program in Group 1. There was no significant improvement in the control group. Conclusion. The balance and quality of life improved significantly after a specific physical therapy program to patients with MS.

Keywords: Physical Therapy. Multiple Sclerosis. Musculoskeletal Equilibrium. Quality of Life.

Citation: Rodrigues IF, Nielson MBP, Marinho AR. Evaluation of the physical therapy on the balance and on the quality of life in multiple sclerosis patients.

\footnotetext{
Trabalho realizado na Escola Superior de Giências da Santa Casa de Misericórdia de Vitória (EMESGAM) e Associação Gapixaba de Portadores de Esclerose Múltipla do Estado do Espírito Santo (ACAPEM-ES).

1. Fisioterapeuta, Pós-graduando em Neurorreabilitação pela EMESCAM - Escola Superior de Ciências da Santa Casa de Misericórdia de Vitória.

2. Fisioterapeuta, Especialista em Fisioterapia Respiratória e Neurofuncional, Doutora, Professora da Disciplina de Saúde do Adulto da EMESCAM.

3. Fisioterapeuta Especialista em Neurologia adulto pela Unicamp, Mestre, Professora e Supervisora da Faculdade Novo Milênio.
}

Endereço para correspondência: Iclaikovski Farias Rodrigues Av. Nossa Senhora da Penha 2190 29045-402 Vitória, ES Fone: (27) 3334-3586 http://www.emescam.com.br E-mail: iclaikovski@ig.com.br

Recebido em: 26/03/08 Revisado em: 27/03/08 a 13/11/08 Aceito em: $14 / 11 / 08$ Conflito de interesses: não 


\section{INTRODUÇÃO}

A Esclerose Múltipla (EM) é principal doença de incapacidade neurológica em adultos jovens e de meia-idade, com manifestações multiformes. Seu curso pode variar de um simples déficit neurológico transitório até a forma mais grave ${ }^{1-3}$. É comumente diagnosticada entre os 20 e 40 anos de idade, sendo que $85 \%$ ocorrem entre os 15 e 50 anos; a maioria das pessoas tem em torno de 30 anos quando é feito o diagnóstico ${ }^{3}$. Predomina nas mulheres, em uma proporção de aproximadamente 2:1, sendo rara em algumas raças (negros africanos e esquimós) e mais encontrada entre brancos de origem norte-européia e mais comum em regiões mais afastadas do Equador ${ }^{3-5}$.

A EM é o principal membro de um grupo de distúrbios conhecidos como doenças desmielinizantes. Apresentam destruição da mielina mediada pelo sistema imunológico, com relativa preservação de outros elementos do tecido nervoso ${ }^{3,4,6}$. As lesões surgem, especialmente, na substância periventricular, no corpo caloso, no trato óptico, cerebelo, tronco e medula ${ }^{3}$. As "placas" são áreas de desmielinização com preservação parcial ou total do axônio, que sofre por disfunção nos canais de $\mathrm{NA}^{+} \mathrm{e}$ da liberação de substâncias neuro-endócrinas, com infiltrado mononuclear nos espaços perivasculares seguidos por gliose e destruição fibrilar dos oligodendrócitos ${ }^{7,8}$. Seu curso é caracterizado pela ocorrência de surtos, definidos como sendo o surgimento de novos sintomas, ou piora súbita de déficits prévios, que tenha duração superior a 24 horas $^{6,9}$. Dois surtos devem ser separados por pelo menos 30 dias de diferença.

A EM pode ser classificada em: EM recidivante, caracterizada por um curso de recidivas recorrentes discretas, entremeadas por períodos de remissão quando a recuperação é completa ou parcial; EM progressiva secundária, depois de um período de recidiva e remissão, a doença entra em uma fase em que há deterioração progressiva, com ou sem recidivas sobrepostas identificáveis; EM progressiva primária, tipificada por déficit neurológico progressivo e cumulativo desde o início ${ }^{10-13}$. Há também, um grupo com um curso progressivo com remissões no qual, ao início progressivo, se sobreporiam surtos com recuperação total ou parcial e outro com uma evolução da doença chamada de benigna, pois os pacientes mantêm longos períodos, até mais de 10 anos, entre o surto e a remissão, embora seja lentamente progressivo ${ }^{3,10,14}$. Seus sinais e sintomas variam em caráter de intensidade e duração, progressão e transição $0^{3,7,10}$. Os déficits funcionais e clínicos se rela- cionam com áreas localizadas de desmielinização no $\mathrm{SNC}^{4}$. Devido à grande variabilidade de localizações anatômicas e seqüência temporal das lesões na EM, as manifestações clínicas da doença variam de um indivíduo para outro ${ }^{1-3}$.

Os principais sintomas motores incluem espasticidade, espasmos reflexos, contraturas, distúrbio da marcha, fadiga, sintomas cerebelares e bulbares como déficit de equilíbrio, nistagmo, tremor intencional, dificuldade de deglutir e respirar ${ }^{6,715}$. Como sintomas sensoriais decorrentes da EM tem-se entorpecimento, parestesia, disestesia, distorção da sensibilidade superficial e dor músculo-esquelética. Os sintomas visuais apresentam-se com diminuição da acuidade, visão dupla, escotolma e dor ocular. O aparecimento súbito de uma neurite óptica, sem qualquer outro sinal ou sintoma do $\mathrm{SNC}$, é frequentemente interpretado como o primeiro sintoma da EM. Quanto os sintomas vesical-intestinais, estes surgem com retenção urinária, incontinência de urgência e/ou aumento da freqüência urinária e constipação. Incluem ainda os sintomas sexuais, caracterizando-se por impotência, diminuição na sensibilidade e lubrificação genital ${ }^{7,10,14,16}$.

A fadiga é uma das manifestações mais freqüentes da EM e pode ser muito debilitante. Exercícios moderados que conservam energia, uso de dispositivos de ajuda, simplificação do trabalho e resfriamento podem ser eficazes para seu controle $\mathrm{e}^{3,12,16}$. A terapêutica farmacológica está voltada para atenuar o processo inflamatório que ocorre durante os surtos e para preveni-los. Para tal são utilizados agentes imunossupressores e imunomoduladores ${ }^{4,7,17}$. Na intervenção fisioterapêutica, terapeuta e paciente trabalham como equipe para minimizar as limitações impostas pela doença, maximizando a capacidade funcional, viabilizando a qualidade de vida em geral e prevenindo complicações debilitantes ${ }^{3}$. A fisioterapia promove qualidade dos padrões do movimento, incentivo ao aprendizado de habilidades motoras, manutenção da força muscular, da coordenação motora e do padrão de marcha, estabilidade postural, bem como um ajuste psicológico entre paciente e família e compreensão dos sintomas da $\mathrm{EM}^{13,18,19}$. As metas em longo prazo de um programa de fisioterapia podem ser as seguintes: melhorar o estado dos sintomas neurológicos da EM detectados à apresentação, melhorar ou manter o nível ótimo de funcionamento físico e psicológico e impedir ou retardar o desenvolvimento de complicações secundárias ${ }^{10,19}$. 
A avaliação dos principais sinais e sintomas da EM torna-se importante, pois quando bem realizada permite uma intervenção mais precoce possível, para minimizar alterações sensório-motoras resultantes de surtos recidivantes, e que podem ser mensuradas de forma tanto objetiva quanto subjetivaa ${ }^{2,7}$. Para quantificar o grau de incapacidade da EM é largamente utilizado por médicos e pesquisadores a Escala de Kurtzke, que freqüentemente determina a eficácia de um tratamento ${ }^{20}$. Outros métodos são consideravelmente importantes na avaliação dos pacientes com EM dentre eles, a Escala de Equilíbrio de Berg ${ }^{21,22}$ para avaliação do equilíbrio e a avaliação da funcionalidade e qualidade de vida, que é feita pela Determinação Funcional da Qualidade de Vida na Esclerose Múltipla (DEFU) ${ }^{8,9,20}$.

Estudos realizados indicam diversos benefícios da intervenção fisioterapêutica em vários aspectos dos pacientes com EM, tornando-se imprescindível avaliar se o processo de reabilitação desses pacientes, melhora a funcionalidade e a qualidade de vida de vida, e minimiza concomitantemente, suas co-morbidades, aumentando sua expectativa de vida ${ }^{18,19}$. O presente estudo, através das avaliações específicas para as alterações de equilíbrio, funcionalidade e qualidade de vida, elaborou e aplicou um protocolo de fisioterapia direcionado com o intuito de promover uma melhora significativa dessas alterações físico-funcionais.

O principal objetivo deste estudo foi avaliar os efeitos da fisioterapia sobre o equilíbrio e a qualidade de vida dos pacientes com EM.

\section{MÉTODO}

\section{Amostra}

O estudo foi do tipo intervencional e analítico, realizado na Associação Capixaba de Portadores de Esclerose Múltipla do Estado do Espírito Santo (ACAPEM-ES), situada no município de Vitória (ES), e na Clínica Escola da Escola Superior de Ciências da Santa Casa de Misericórdia de Vitória - EMESCAM.

A presente pesquisa foi submetida à apreciação e aprovação do Comitê de Ética e Pesquisa da Escola Superior de Ciências da Santa Casa de Mi- sericórdia de Vitória - EMESCAM, sob o protocolo de $\mathrm{n}^{\circ}$ 069/2007. Todos os pacientes foram voluntários e assinaram termo de consentimento.

Vinte portadores de Esclerose Múltipla de ambos os sexos foram aleatorizados em 2 grupos de 10 indivíduos: Grupo 1: indivíduos submetidos a 15 intervenções fisioterapêuticas específicas para EM, e Grupo 2: indivíduos que não participaram do programa específico, entretanto, realizaram fisioterapia convencional (uso de bastões, caminhadas, analgesias e condicionamento cardiorrespiratório e neuromuscular) uma vez por semana, totalizando 8 intervenções.

Foram incluídos os indivíduos com condições de responder os questionários e realizar as atividades do estudo. Foram excluídos os pacientes cadeirantes e que apresentaram déficit cognitivo, problemas visuais e auditivos, que os impossibilitaram de participar ativamente da pesquisa.

Quinze 15\% (3) dos indivíduos eram do sexo masculino e $85 \%$ (17) do sexo feminino, com média de idade de $40 \pm 12$ anos, variando entre 18 a 63 anos. Participaram da pesquisa pacientes com qualquer tipo de EM, não observado um tipo específico mediante diagnóstico clínico.

\section{Avaliação}

Os participantes foram submetidos às avaliações do equilíbrio, por meio da Escala de Equilíbrio de Berg, e da qualidade de vida, através da DEFU (Escala de Determinação Funcional da Qualidade de Vida em indivíduos com EM), antes e após a intervenção fisioterapêutica.

\section{Intervenção}

\section{Treinamento Especifico}

Treinamento Específico proposto pelos autores, com base na literatura.

Foram utilizados como instrumentos de trabalho cama-elástica, barra-paralela, prancha de desequilíbrio. O programa de treinamento específico consistiu de técnicas de treino de equilíbrio em bola suíça, "cama-elástica", prancha de desequilíbrio, treino de marcha na barra paralela, circuitos, rampa e escada, e fortalecimento dos grupos musculares de

Tabela 1. Valores descritivos referentes à Escala de Equilíbrio de Berg antes e depois da intervenção.

\begin{tabular}{|l|c|c|c|c|c|c|}
\hline \multirow{2}{*}{ Período } & \multicolumn{3}{|c|}{ Grupo 1 } & \multicolumn{3}{c|}{ Grupo 2 } \\
\cline { 2 - 7 } & Média & DP & Mediana & Média & DP & Mediana \\
\hline Antes da intervenção & 46,6 & 11,6 & 53,0 & 33,5 & 14,1 & 35,0 \\
\hline Após intervenção & 48,2 & 10,3 & 54,0 & 33,7 & 13,9 & 34,5 \\
\hline
\end{tabular}


Tabela 2. Valores descritivos referentes à funcionalidade antes e depois da intervenção.

\begin{tabular}{|l|c|c|c|c|c|c|}
\hline \multirow{2}{*}{ Período } & \multicolumn{3}{|c|}{ Grupo 1 } & \multicolumn{3}{c|}{ Grupo 2} \\
\cline { 2 - 7 } & Média & DP & Mediana & Média & DP & Mediana \\
\hline Antes da intervenção & 100,6 & 29,8 & 94,0 & 97,9 & 18,3 & 95,5 \\
\hline Após intervenção & 104,8 & 29,0 & 99,5 & 97,6 & 17,9 & 96,5 \\
\hline
\end{tabular}

MMII. As atividades foram realizadas 3 (três) vezes por semana (segundas, quartas e sextas feiras) no período matutino, na Clínica Escola de Fisioterapia da EMESCAM, com o seguinte protocolo:

No primeiro dia, foi realizado trabalho de equilíbrio em bola suíça e cama-elástica, (objetivo dar propriocepção e desequilíbrio ao paciente) solicitando isometria de MMSS, tronco e MMII, durante 40 minutos, com intervalos para não sobrecarregar e fadigar o paciente. No segundo dia, trabalhou-se o equilíbrio sem carga, em rampa, cama-elástica, e pista (circuito), com listas, transpassando objetos e obstáculos de alturas variadas, também durante 40 minutos. No terceiro dia, trabalho com exercícios de Frenkel, realizados com a parte sustentada ou não, uni ou bilateralmente, praticados em movimentos contínuos e sincronizados, de forma lenta e equilibrada, em quatro posições básicas: deitado, sentado, em pé e deambulando, progredindo desde posturas de maior estabilidade até posturas de maior desafio. Estimulou-se a concentração e a repetição dos movimentos, bem como a realização dos exercícios com os olhos fechados.

Exemplos dos exercícios de Frenkel:

1. Decúbito dorsal: Flexão dos quadris e joelhos e extensão de cada membro, pés apoiados totalmente numa base.

2. Abdução dos quadris e adução de cada membro com os pés apoiados, joelhos flexionados: e a seguir, estendidos.

3. Flexão dos quadris e joelhos e extensão de cada membro, com os calcanhares erguidos da base.

4. Calcanhar de um dos membros até a perna oposta (dedos, tornozelo, tíbia e patela).

5. Calcanhar de um dos membros até o joelho oposto, deslizando na crista da tíbia abaixo, até o tornozelo.

6. Flexão dos quadris e joelhos, e extensão de ambos os membros, pernas juntas.

7. Movimentos recíprocos de ambos os membros: flexão de uma perna durante a extensão da outra.

8. Sentado: extensão do joelho e flexão da cada membro: progrida fazendo marcação de tempo.

9. Abdução e adução dos quadris.
10. Posicionamento alternado dos pés até um alvo específico (usando-se marcações no piso, ou uma grade).

11. Levantar e sentar: sob uma contagem específica.

12. Em pé: posicionamento do pé até um alvo especificado (marcações no piso uma grade).

13. Transferência de peso.

14. Andando: De lado ou para a frente, sob uma contagem especificada (um colchonete de Frenkel, linhas paralelas, ou marcações no piso podem ser usados como alvos para o controle do posicionamento dos pés, comprimento das passadas e largura dos passos).

15. Girar, sob uma contagem específica (as marcas no piso podem ter utilidade na manutenção de uma base estável de sustentação).

\section{Fisioterapia Convencional}

Na Fisioterapia Convencional, foi adotado medidas para se trabalhar com os mesmos objetivos, com barras, bolas e obstáculos, porém sem ênfase no protocolo proposto com base na literatura que relata os exercícios de Frenkel.

\section{Análise estatística}

Foi realizada análise descritiva dos dados, através de tabelas com média, desvio padrão e mediana para a Escala de Equilíbrio de Berg e DEFU. As médias dos grupos antes e após a intervenção foram testadas através do teste t de Student pareado. O pacote estatístico SPSS 15 - Social Package Statistical Science - foi utilizado nesta análise.

\section{RESULTADOS}

Mediante a Escala de Equilíbrio de Berg, obtiveram-se variações significativas entre os indivíduos. Dos 10 sujeitos que realizaram o protocolo proposto (Grupo 1), 6 (60\%) apresentaram equilíbrio bom, $3(30 \%)$ apresentaram equilíbrio regular e $1(10 \%)$ equilíbrio ruim. Uma porcentagem razoável de indivíduos $(56 \%)$ apresentou risco para quedas. A funcionalidade e a qualidade de vida demonstraram que $60 \%$ dos indivíduos estavam abaixo do nível esperado para qualidade de vida, onde os itens mais 
Tabela 3. Dados demográficos, valores da Escala de Equilíbrio de Berg e valores da Escala de Determinação Funcional da Qualidade de Vida (DEFU) para os pacientes com Esclerose Múltipla do grupo estudo e do grupo controle, antes e depois da intervenção.

\begin{tabular}{|c|c|c|c|c|c|c|}
\hline Grupo & Paciente (sexo) & Idade & valores Berg antes & Valores Berg depois & DEFU Antes & DEFU depois \\
\hline \multirow[t]{10}{*}{ Estudo (Grupo 1) } & $1 \mathrm{~F}$ & 18 & 56 & 58 & 51 & 58 \\
\hline & $2 \mathrm{~F}$ & 22 & 42 & 46 & 42 & 50 \\
\hline & $3 \mathrm{M}$ & 41 & 56 & 60 & 66 & 68 \\
\hline & $4 \mathrm{~F}$ & 41 & 52 & 58 & 61 & 66 \\
\hline & $5 \mathrm{M}$ & 18 & 52 & 59 & 91 & 99 \\
\hline & $6 \mathrm{~F}$ & 30 & 54 & 62 & 109 & 109 \\
\hline & $7 \mathrm{~F}$ & 41 & 53 & 57 & 142 & 144 \\
\hline & $8 \mathrm{~F}$ & 51 & 53 & 60 & 149 & 152 \\
\hline & $9 \mathrm{~F}$ & 49 & 39 & 41 & 62 & 68 \\
\hline & $10 \mathrm{M}$ & 48 & 53 & 62 & 86 & 86 \\
\hline \multirow[t]{10}{*}{ Controle (Grupo 2) } & $1 \mathrm{~F}$ & 43 & 53 & 53 & 63 & 66 \\
\hline & $2 \mathrm{M}$ & 48 & 48 & 50 & 78 & 76 \\
\hline & $3 \mathrm{~F}$ & 37 & 36 & 38 & 97 & 97 \\
\hline & $4 \mathrm{~F}$ & 35 & 56 & 56 & 88 & 88 \\
\hline & $5 \mathrm{~F}$ & 49 & 18 & 18 & 86 & 88 \\
\hline & $6 \mathrm{~F}$ & 63 & 13 & 13 & 87 & 91 \\
\hline & $7 \mathrm{~F}$ & 46 & 49 & 51 & 79 & 79 \\
\hline & $8 \mathrm{~F}$ & 36 & 54 & 56 & 77 & 78 \\
\hline & $9 \mathrm{~F}$ & 24 & 16 & 19 & 46 & 49 \\
\hline & $10 \mathrm{~F}$ & 32 & 28 & 31 & 153 & 156 \\
\hline
\end{tabular}

$\mathrm{F}=$ feminino; $\mathrm{M}=$ masculino.

comprometidos foram o déficit de equilíbrio, o estado emocional e o convívio social. Nos indivíduos do grupo controle, não foi observado melhora significativa quanto ao equilíbrio e funcionalidade.

A comparação da média do equilíbrio de Berg, do Grupo 1, antes da intervenção foi de 46,6 $\pm 11,6$ e após de 48,2 $\pm 10,3$ (Tabela 1). O teste t pareado evidenciou uma melhora estatisticamente significante do equilíbrio após a intervenção $(p=0,011)$. Já no grupo controle não houve melhora significativa: 33,5 antes e 33,7 após a intervenção $(\mathrm{p}=0,591)$ (Tabela 2).

A comparação da média da Funcionalidade, do Grupo 1, antes da intervenção foi de 100,6 $\pm 29,8$ e após de 104,8 $\pm 29,0$ (Tabela 2). Este resultado indica que houve uma melhora significante da funcionalidade e qualidade de vida dos indivíduos com $\mathrm{EM}(\mathrm{p}=0,006)$. Já no grupo controle não houve diferença estatística nessas médias, 97,9 antes e 97,6 após $(\mathrm{p}=0,697)$.

\section{DISCUSSÃO}

A amostra foi composta predominantemente pelo sexo feminino $(85 \%)^{11,16}$, sendo mais comum duas ou três vezes em mulheres ${ }^{3,13}$. A média de idade encontrada nos indivíduos do presente estudo correlaciona com estudos epidemiológicos ${ }^{2,5,8,15}$. O diagnóstico da EM é comumente realizado entre 15 e $50 \operatorname{anos}^{5,7,14}$.
Na pesquisa realizada, o equilíbrio se apresentou bom em mais da metade dos sujeitos, como em estudo realizado por Mendes et al..$^{16} \mathrm{e}$ Myamoto et al..$^{21}$, que avaliaram portadores de EM e observou que o risco de quedas nestes indivíduos associava-se ao sexo, medo de cair, curso da doença e uso de cadeiras de rodas ${ }^{22}$.

Foi verificado que a maior queixa dos pacientes com EM era o desequilíbrio, evidenciando a disfunção vestibular na maioria dos $\operatorname{casos}^{13,22}$.

Optou-se por avaliar a funcionalidade e qualidade de vida através da DEFU por ser utilizada como instrumento de confiabilidade sensibilidade e validade ${ }^{20}$.

A funcionalidade e a qualidade de vida se apresentaram abaixo do nível esperado para qualidade de vida ${ }^{9,16}$. A diminuição da capacidade aeróbica, da composição corporal e força são fatores que causam impacto negativo na qualidade de vida ${ }^{6,13,18}$. A fisioterapia tem-se mostrado eficaz na melhora do equilíbrio e qualidade de vida ${ }^{19}$ (Tabela 3 ).

No entanto, pesquisas sobre os efeitos da fisioterapia e atividade fisica evidenciando a melhora da qualidade de vida nesses pacientes devem ser realizadas e incentivadas. Um número crescente dos estudos mostrando efeitos benéficos do exercício nos portadores de EM, e que tais programas não são somente para melhorar parâmetros de aptidão, mas realçar a qualidade de vida e aumentar o potencial neuroprotetor ${ }^{9,18,19}$. 
A presente pesquisa foi importante para avaliar e tratar os pacientes com EM viabilizando e fundamentando um possível protocolo de tratamento direcionado às alterações específicas encontradas.

Torna-se, portanto, necessário o incentivo à pesquisa dos efeitos benéficos da fisioterapia para esses pacientes para traçar e implantar um protocolo de tratamento direcionado aos sinais e sintomas apresentados, visando mais funcionalidade e bem-estar.

\section{CONCLUSÃO}

Conclui-se que houve melhora do equilíbrio, apesar dos sujeitos apresentarem risco para quedas, houve melhora da funcionalidade apesar indivíduos estarem abaixo do nível esperado para qualidade de vida, que o acompanhamento de pacientes com EM pela fisioterapia direcionada as alterações específicas promove mais funcionalidade e qualidade de vida.

\section{REFERÊNCIAS BIBLIOGRÁFICAS}

1. Frankel D. Esclerose Múltipla. In: Umpred DA. Reabilitação neurológica. São Paulo: Manole, 2004, 627-47.

2. Desouza L, Bates D, Moran G. Esclerose Múltipla. In: Cash MS. Neurologia para fisioterapeutas. São Paulo: Premier, 2000, 149-65.

3. Callegaro D. Esclerose Múltipla. In: Nitrini R, Bacheschi LA. A neurologia que todo médico deve saber. São Paulo: Atheneu, 2003, 335-40.

4. Thompson A, Skinner A, Piercy J. Doenças do cérebro e da medula espinal. In: Fisioterapia de Tidy. São Paulo: Santos, 2002, 331-3.

5. Oliveira EML, Annes M, Oliveira ASB, Gabbai AA. Estudo clínico de 50 pacientes acompanhados no ambulatório de neurol ogia UNIFESP-EPM. Arq Neuropsiquiatr 1999;57(1):55-7.

6. Grzesiuk AK. Características clínicas e epidemiológicas de 20 pacientes portadores de esclerose múltipla acompanhados em Cuiabá

- Mato Grosso. Arq Neuropsiquiatr 2006;64(3A):635-8.
7. O'Sullivan SB. Esclerose Múltipla. In: O'Sullivan SB, Schmitz TS. Fisioterapia avaliação e tratamento. 2a ed. São Paulo: Manole, 1993, 527-48. 8. Nogueira TM, Santos-Filho SD. Proposta de fisioterapia comunitária em pacientes portadores de esclerose múltipla. Rev Reab 2002;14:20-5.

9. Fisk JD, Ritvo PG, Ross L, Haase DA, Marrie TJ, Schlech WF. Measuring the functional inpact of fatigue: initial validation of the impact scale. Clin Inf Dis 1994;18(1):12-22.

10. Edwards S. Tratamento a longo prazo para pacientes com incapacidade residual ou progressiva. In: Fisioterapia neurológica - uma abordagem centrada na revolução de problemas. Porto Alegre: Artmed, 1999, 207-11.

11. Silva JA, Cohen BA. Esclerose múltipla. In: Delisa JA, Gans BM.

Tratado de medicina de reabilitação - princípios e práticas. 3. ed. Vol 2. São Paulo: Manole, 2002, 1308-12.

12. Tabosa A, Yamamura Y. Fadigas como fator etiopatogênico energético. Rev Paul Acup 2000;6(2):101-4.

13. Vargas AL, Morais RCA, Cunha MCB. Exercícios terapêuticos para portadores de esclerose múltipla com déficitde coordenação motora e equilíbrio. Fisioter Bras RJ 2000;3(3):151-6.

14. Vecino MC, Haussen SR. Esclerose Múltipla. Rev Med Santa Casa 1999;10(17):1820:35.

15. Miller JR. Esclerose Múltipla. In: Rowland LP, Merrit MD. Tratado de neurologia. 10.ed. São Paulo: Guanabara e Koogan, 2002, 670-86.

16. Mendes MF, Tilbery CP, Felipe E. Fadiga e esclerose múltipla - estudo preliminar de 15 casos através de escalas de auto-avaliação. Arq Neuropsiquiatr 2000;58(2):467-70.

17. Melaragno RF. Causas da EM - aspectos imunológicos. In: Esclerose Múltipla - manual para pacientes e suas famílias. São Paulo: Abem, 1992, 41-52.

18. Heesen C, Romberg A, Gold A, Schulz KH. Physical exercise in multiple sclerosis: supportive care or a putative disease- modifying treatment. Exp Rev Neurother 2006;6(3):347-55

19. Motl RW, Morris KS, Hu L, Doerksen SE, Elavsky S, Konopack JF. Enhancing physical activity adherence and well-being in multiple sclerosis: a randomised controlled trial. Rev Citeulike Mult scler 2007;13(5):652-9.

20. Kurtzke JF. Rating neurologic impairment in mutiple sclerosis: an expanded disability status scale (EDSS). Ann NY Acad Sci Neurology 1983;33(11):1444-52.

21. Myamoto ST, Lombardi Junior I, Berg KO, Ramos LR, Natour $\mathrm{J}$. Brazilian version of the Berg balance scale. Braz J Med Biol Res 2004;37(9):1411-21.

22. Berg KO, Wood-Dauphnée S, Willians JI. The balance scale: reability assessment with elderly residents and patients with an acute stroke. Scand J Rehab Med 1995;10(4):27-36. 